\title{
Gesamtinhaltsverzeichnis 2016
}

DOI 10.1515/mgzs-2016-0142

\section{Aufsätze}

Philip Haas

"Si vis societatem para ad militiam." Machiavellis Rezeption und

Weiterentwicklung des Vegetius — 1

Holger Afflerbach

Kronprinz Rupprecht von Bayern im Ersten Weltkrieg - 21

Chris Helmecke

Ein wanderer« Oberbefehlshaber? Generaloberst Rudolf Schmidt und die deutsche Besatzungsherrschaft in der Sowjetunion 1941-1943 55

\section{Gaj Trifković}

Carnage in the Land of Three Rivers: The Syrmian Front 1944-1945 94

Oliver Stein

„Orientfahrten«. Deutsche Soldaten im Osmanischen Reich und der Krieg als Reiseerlebnis 1914 bis $1918-327$

\section{Christian Götter}

Von der militärischen Maßnahme zum politischen Machtmittel. Die Entwicklung der Wirtschaftsblockade im Ersten Weltkrieg — 359

\section{Mathias Schütz}

Kein Vergessen. Die Europa-Föderalisten, der Verband deutscher Soldaten und die europäischen Veteranentreffen 1952/53 388

\section{Literaturbericht}

Malte König

Krieg und Militär in der ersten Hälfte des 20. Jahrhunderts: Tendenzen und Leerstellen der deutschsprachigen Italienforschung 1970 bis $2015-415$ 


\section{Nachrichten aus der Forschung}

Karen Bruhn

„Kiel und die Marine 1865-2015. 150 Jahre gemeinsame Geschichte»

Sven Wunderlich

„Besatzungskinder und Wehrmachtskinder - Auf der Suche nach Identität und Resilienz« 128

Bernhard Bachinger und Richard Lein

"Globaler Krieg. Visionen und ihre Umsetzung" — 134

Felicitas Söhner und Deborah Frommeld

"Nachkrieg und Medizin in Deutschland im 20. Jahrhundert» — 141

Bastian Matteo Scianna

„Gewaltkulturen von den Kolonialkriegen bis zur Gegenwart.» — 145

Karen Bähr

„Der Krieg ist vorbei. Heimkehr - Trauma - Weiterleben« — 151

Cornelia Grosse und Leonie Hieck

"Geschichtsbewusstsein als soldatische Kernkompetenz.

60 Jahre Historische Bildung in der Bundeswehr« - 157

Ulrich Hertel

„Der Wiener Kongress und seine Folgen. Großbritannien, Europa

und der Frieden im 19. und 20. Jahrhundert« - 435

Anja Kircher-Kannemann

"Zeit und Militär in der Frühen Neuzeit» — 442

Ringo Müller

»Militärisches Wissen vom 16. bis zum 19. Jahrhundert« — 445

Stephan Jaeger

"Museums, Memorials, and War« -450 
Lukas Boser und Philippe Müller

„Pulverdampf und Kreidestaub. Wissenstransfer zwischen Schweizer Militär

und Schweizer Schule« — 456

Robert Fahr

»Burg, Stadt und Kriegführung im 17. Jahrhundert» - 461

Martin Jost

»Kindheit im Zweiten Weltkrieg. Eine vergleichende Perspektive« - 467

Denis Strohmeier

"Politische Vorstellungen vom deutschen Soldaten nach 1945« 474

Claudia Kemper

»Fenster im Kalten Krieg. Über Grenzen, Alternativen und

Reichweite einer binären Ordnungsvorstellung" — 481

Yves Schmitz

"Krieg und organisierte Gewalt im Computerspiel -

Militärhistorische Narrative, Räume und Geschichtsbilder« — 489

\section{Buchbesprechungen}

Heinz Stübig

1813 im europäischen Kontext. Hrsg. von Birgit Aschmann

und Thomas Stamm-Kuhlmann — 541

Alaric Searle

Norman Ächtler, Generation in Kesseln. Das Soldatische Opfernarrativ im westdeutschen Kriegsroman 1945-1960 - 300

Horst Boog ( $($ )

Hans-Werner Ahrens, Die Luftbrücke nach Sarajevo 1992 bis 1996.

Transportflieger der Luftwaffe und der Jugoslawienkrieg

Michael Epkenhans

Jürgen Angelow, Der Weg in die Urkatastrophe. Der Zerfall

des alten Europa 1900-1914 — 241 


\section{Torsten Diedrich}

Philipp Aumann, Rüstung auf dem Prüfstand. Kummersdorf, Peenemünde und die totale Mobilmachung. Hrsg. vom Historisch-Technischen Museum Peenemünde -521

Andreas R. Hofmann

Richard Bassett, For God and Kaiser. The Imperial Austrian Army, 1619 to $1918-502$

\section{Günther Kronenbitter}

Biographisches Handbuch des deutschen Auswärtigen Dienstes 1871-1945, Bd 4: S. Bearb. von Bernd Isphording, Gerhard Keiper und Martin Kröger 509

Günther Kronenbitter

Biographisches Handbuch des deutschen Auswärtigen Dienstes 1871-1945, Bd 5: T-Z, Nachträge. Bearb. von Bernd Isphording, Gerhard Keiper und Martin Kröger — 509

\section{Ludger Tewes}

Ralf Blank, Ruhrschlacht. Das Ruhrgebiet im Kriegsjahr $1943-276$

\section{Frank Ganseuer}

Peter Blickle, Der Bauernjörg. Feldherr im Bauernkrieg. Georg Truchsess von Waldburg 1488-1531 193

Dieter Hartwig

Bernd Bölscher, Hitlers Marine im Landkriegseinsatz.

Eine Dokumentation -601

Heinz Stübig

Klaus-Jürgen Bremm, Die Schlacht. Waterloo 1815

Martin Rink

Franz Brendle, Das konfessionelle Zeitalter — 526

Max Plassmann

Norbert Büllesbach, Aus dem Rheinland in den Krieg. Mit einem rheinischen Infanterie-Regiment auf den Schlachtfeldern des Ersten Weltkriegs 
Christian Koller

Ian Buruma, '45: Die Welt am Wendepunkt

Jochen Maurer

Rainer Buske, Kunduz: Ein Erlebnisbericht über einen militärischen Einsatz der Bundeswehr in Afghanistan im Jahre $2008-653$

Björn Hofmeister

Brian E. Crim, Antisemitism in the German Military Community and the Jewish Response, 1914-1938 — 257

Christian Streit

Deutsche Berichte aus dem Osten 1942-1943. Dokumente der Einsatzgruppen in der Sowjetunion, Bd 3. Hrsg. von Klaus-Michael Mallmann [u.a.] - 612

\section{Christian Streit}

Deutsche Besatzungsherrschaft in der UdSSR 1941-1945. Dokumente der Einsatzgruppen in der Sowjetunion, Bd 2. Hrsg. von Andrej Angrick [u.a.] - 612

Gabriele Bosch

Die Dienstbibliothek des Brandenburg-Preußischen Hausarchivs.

Katalog, bearb. von Herzeleide Henning — 167

Volker Koop

William Durie, The United States Garrison Berlin 1945-1994

"Mission Accomplished», book $1-315$

Michael Peters

Richard van Emden, Meeting the Enemy. The Human Face

of the Great War — 560

\section{Georg Wurzer}

Erinnerung an Diktatur und Krieg. Brennpunkte des kulturellen Gedächtnisses zwischen Russland und Deutschland seit 1945. Hrsg. von Andreas Wirsching [u.a.] — 648

Sven Lange

Urte Evert, Die Eisenbraut. Symbolgeschichte der militärischen Waffe von 1700 bis 1945 172 
Günther Kronenbitter

Marvin Benjamin Fried, Austro-Hungarian War Aims in the Balkans during World War I -576

Stephan Theilig

Frieden übersetzen in der Vormoderne. Translationsleistungen in Diplomatie, Medien und Wissenschaft. Hrsg. von Heinz Duchhardt und Martin Espenhorst — 197

Andreas Himmelsbach

Miriam Gebhardt, Als die Soldaten kamen. Die Vergewaltigung deutscher Frauen am Ende des Zweiten Weltkriegs — 287

Bruno Thoß

Stephan Geier, Schwellenmacht. Bonns heimliche Atomdiplomatie von Adenauer bis Schmidt — 305

Martin Meier

Axel Gotthard, Der liebe vnd werthe Fried. Kriegskonzepte und Neutralitätsvorstellungen in der Frühen Neuzeit — 199

\section{Rolf Hosfeld}

Jürgen Gottschlich, Beihilfe zum Völkermord. Deutschlands Rolle bei der Vernichtung der Armenier 245

Heather Jones

The Great War and Veterans' Internationalism. Ed. by Julia Eichenberg and John Paul Newman — 588

Martin Moll

Thomas R. Grischany, Der Ostmark treue Alpensöhne. Die Integration der Österreicher in die großdeutsche Wehrmacht, 1938-45 — 596

Martin Meier

Elisabeth Großegger, Mythos Prinz Eugen. Inszenierung

und Gedächtnis — 208

Jochen Maurer

Uwe Hartmann, War Without Fighting? The Reintegration of Former Combatants in Afghanistan seen through the Lens of Strategic Thougt -657 
Max Plassmann

Historiker und Archivar im Dienste Preußens. Festschrift für Jürgen Kloosterhuis. Hrsg. von Hans-Christof Kraus und Frank-Lothar Kroll — 505

Martin Moll

Lothar Höbelt, „Stehen oder Fallen?« Österreichische Politik

im Ersten Weltkrieg — 573

Hiram Kümper

Martin Hofbauer, Vom Krieger zum Ritter. Die Professionalisierung der bewaffneten Kämpfer im Mittelalter — 189

Heiko Herold

Jörg-Michael Hormann und Eberhard Kliem, Die Kaiserliche Marine im Ersten Weltkrieg. Von Wilhelmshaven nach Scapa Flow — 243

Jens Westemeier

Esther-Julia Howell, Von den Besiegten lernen? Die kriegsgeschichtliche

Kooperation der U.S. Armee und der ehemaligen Wehrmachtselite

1945-1961 - 636

Gabriele Bosch

Florian Huber, Kind, versprich mir, dass du dich erschießt. Der Untergang der kleinen Leute $1945-291$

Martin Rink

Humanitarian Intervention: A History. Ed. by Brendan Simms and D.J.B. Trim — 498

Christoph Nübel

Das Imaginäre des Kalten Krieges. Beiträge zu einer Kulturgeschichte des OstWest-Konfliktes in Europa. Hrsg. von David Eugster und Sibylle Marti — 624

Andreas R. Hofmann

Leighton S. James, Witnessing the Revolutionary and Napoleonic Wars in German Central Europe — 535

Peter A. Popp

Konrad H. Jarausch, Out of Ashes. A New History of Europe

in the Twentieth Century — 517 


\section{Martin Rink}

Just and Unjust Military Intervention. European Thinkers from Vitoria to Mill. Ed. by Stefano Recchia and Jennifer M. Welsh — 498

\section{Marius Kettmann}

Manfred Kanetzki, MiGs über Peenemünde: Die Geschichte der NVA-Fliegertruppenteile auf Usedom - 309

\section{Andreas R. Hofmann}

Matthias Kaufmann, "... die merkwürdigsten fremden Truppen.» Die Wahrnehmung der Baschkiren in den Napoleonischen Kriegen 1812-1814 im Spiegel zeitgenössischer Berichte - 539

\section{Andreas R. Hofmann}

Catriona Kennedy, Narratives of the Revolutionary and Napoleonic Wars. Military and Civilian Experience in Britain and Ireland - 535

Andreas R. Hofmann

Jürgen Kloosterhuis, Menzel militaris. Sein »Armeewerk« und das »Leuthen«-Bild im militärhistorischen Quellenkontext — 551

Bernd Jürgen Wendt

Jobst C. Knigge, Angst vor Deutschland - Mussolinis Deutschlandbild - $\mathbf{5 9 0}$

\section{Loretana de Libero}

Königin Luise von Preußen. Briefe und Aufzeichnungen 1786-1810. Mit einer Einl. von Hartmut Boockmann hrsg. von Malve Gräfin Rothkirch — 530

Martin Meier

Konrad Kramar und Georg Mayrhofer, Prinz Eugen. Heros und Neurose — 208

Harald Potempa

Mario Kramp, 1914: Vom Traum zum Albtraum. Köln und der Beginn des Bombenkriegs in Europa — 563

\section{Christian Koller}

Jonas Kreienbaum, „Ein trauriges Fiasko«. Koloniale Konzentrationslager im südlichen Afrika 1900-1908 — 554 
Martin Moll

Gerd Krumeich, Deutschland, Frankreich und der Krieg. Historische Studien zu Politik, Militär und Kultur. Hrsg. von Susanne Brandt, Thomas Gerhards und Uta Hinz 182

Rüdiger Overmans

Lager vor Auschwitz. Gewalt und Integration im 20. Jahrhundert. Hrsg. von Christoph Jahr und Jens Thiel — 513

Benjamin Hasselhorn

Max Lehmann, Bismarck. Eine Charakteristik. Hrsg. von Gertrud Lehmann 236

Janine Rischke-Neß

Steffen Leins, Das Prager Münzkonsortium 1622/23. Ein Kapitalgeschäft im Dreißigjährigen Krieg am Rande der Katastrophe - 205

Christian Koller

Loretana de Libero, Rache und Triumph. Krieg, Gefühle und Gedenken in der Moderne -264

Rüdiger Wenzke

Hans-Georg Löffler, Bernd Biedermann und Wolfgang Kerner, Paraden und Rituale der NVA 1956-1990 - 646

Max Plassmann

Ulrich March, Grundzüge der Militärgeschichte. Krieg und politische Kultur — 165

Heiko Herold

Maritime Sicherheit. Hrsg. von Sebastian Bruns, Kerstin Petretto und David Petrovic — 317

\section{Christian Koller}

Mass Killings and Violence in Spain, 1936-1952. Grappling with the Past. Ed. by Peter Anderson and Miguel Ángel del Arco Blanco — 265 


\section{Martin Moll}

Materialschlachten. Der Erste Weltkrieg und seine Darstellungsressourcen in Literatur, Publizistik und populären Medien 1899-1929. Hrsg. von Christian Meierhofer und Jens Wörner 557

\section{Hiram Kümper}

The Medieval Way of War. Studies in Medieval Military History in Honor of Bernard S. Bachrach. Ed. by Gregory I. Halfond — 191

\section{Max Plassmann}

Militär und Gesellschaft in Preußen. Quellen zur Militärsozialisation 1713-1806. Archivalien in Berlin, Dessau und Leipzig. Teil 1, Bd 1: Geheimes Staatsarchiv Preußischer Kulturbesitz (1. Hälfte); Teil 1, Bd 2: Geheimes Staatsarchiv Preußischer Kulturbesitz (2. Hälfte); Teil 2: Weitere Archive, Bibliotheken und Museen in Berlin, Dessau und Leipzig; Teil 3: Indices und Systematiken. Hrsg. von Jürgen Kloosterhuis [u.a.] — 212

Andreas R. Hofmann

Militär und Mehrsprachigkeit im neuzeitlichen Europa. Hrsg. von Helmut Glück und Mark Häberlein — 202

\section{Heiko Herold}

Ernst Dietrich Baron von Mirbach, Prinz Heinrich von Preußen. Eine Biographie des Kaiserbruders 238

\section{Günther Kronenbitter}

Martin Moll, Die Steiermark im Ersten Weltkrieg. Der Kampf des Hinterlandes ums Überleben 1914-1918 — 581

\section{Marie Tritsch}

Mythes et tabous des relations franco-allemandes au XXe siècle. / Mythen und Tabus der deutsch-französischen Beziehungen im 20. Jahrhundert. Hrsg. von/ed.par Ulrich Pfeil — 185

\section{Frank Reichherzer}

Günter Nagel, Wissenschaft für den Krieg. Die geheimen Arbeiten der Abteilung Forschung des Heereswaffenamtes -273

Heinz Stübig

Napoleon on War. Ed. by Bruno Colson - 217 
Emilie Terre

Sergej Nelipovič, Krovavyj oktjabr' 1914 goda -248

Martin Moll

1945 - Ikonen eines Jahres. 108 Photographien von 42 Photographen. Mit einem Einführungstext von Norbert Frei. Hrsg. von Lothar Schirmer — 296

Martin Moll

1945 - Niederlage. Befreiung. Neuanfang. Zwölf Länder Europas nach dem Zweiten Weltkrieg. Hrsg. vom Deutschen Historischen Museum — 296

Martin Moll

1945 - Niederlage und Neubeginn. Hrsg. von Ernst Piper 296

Christopher Theel

NS-Militärjustiz im Zweiten Weltkrieg. Disziplinierungs- und Repressionsinstrument in europäischer Dimension. Hrsg. von Claudia Bade, Lars Skowronski und Michael Viebig — 604

Andreas R. Hofmann

Frank Palmowski, Die Belagerung von Erfurt 1813/14 -545

Heinz Stübig

Peter Paret, Clausewitz in His Time. Essays in the Cultural and Intellectual History of Thinking about War -532

André Müllerschön

Pierluigi Pironti, Kriegsopfer und Staat. Sozialpolitik für Invaliden, Witwen und

Waisen des Ersten Weltkriegs in Deutschland und Italien (1914-1924) — 584

Georg Wurzer

Pis'ma s vojny 1914-1917. Hrsg. von A.B. Astašov und P.A. Simmons - 251

Markus Pöhlmann

Wolfram Pyta, Hitler. Der Künstler als Politiker und Feldherr.

Eine Herrschaftsanalyse -268

Martin Moll

Manfried Rauchensteiner und Josef Broukal, Der Erste Weltkrieg und das Ende der Habsburgermonarchie 1914-1918. In aller Kürze — 579 
Thomas Beddies

Karl-Heinz Reger, „Dann sprang er über Bord». Alltagspsychologie und psychische Erkrankung an Bord britischer Schiffe im 19. Jahrhundert

Andreas R. Hofmann

Revisiting Napoleon's Continental System. Local, Regional and European

Experiences. Ed. by Katherine B. Aaslestad and Johan Joor — 219

Winfried Heinemann

Stefan Rosenträger, Die NS-Zeit im Geschichts- und Traditionsverständnis der Bundeswehr. Eine Auswertung ihrer periodischen Veröffentlichungen (1955-1972) - 641

Marcus von Salisch

Sebastian Schaar, Wahrnehmungen des Weltkrieges. Selbstzeugnisse

Königlich Sächsischer Offiziere 1914 bis 1918 — 254

Martin Rink

Sibylle Scheipers, Unlawful Combatants. A Genealogy of

the Irregular Fighter -495

Gerhard Krebs

Klaus Scherer, Nagasaki. Der Mythos der entscheidenden Bombe 620

Harald Potempa

Veit Scherzer, Das Auszeichnungssystem der Wehrmacht 593

Frank Ganseuer

Rüdiger Schiel, Die vergessene Partnerschaft. Kaiserliche Marine und k.u.k. Kriegsmarine 1871-1914 566

Rüdiger Overmans

Barbara Schmitter Heisler, From German Prisoner of War to American Citizen. A Social History with 35 Interviews - 303

Heinz Stübig

Karin Schneider und Eva Maria Werner, Europa in Wien. Who is who beim Wiener Kongress 1814/15 548 


\section{Christian Koller}

Jürg Schoch, "Mit Aug' und Ohr für's Vaterland!« Der Schweizer Aufklärungsdienst von Heer \& Haus im Zweiten Weltkrieg — 285

\section{Rolf Steininger}

Victor Sebestyen, 1946. Das Jahr, in dem die Welt neu entstand 632

Helmut R. Hammerich

Secret Intelligence in the European States System, 1918-1989.

Ed. by Jonathan Haslam and Karina Urbach — 519

\section{Alexander Querengässer}

Marco Sigg, Der Unterführer als Feldherr im Taschenformat. Theorie und Praxis der Auftragstaktik im deutschen Heer 1869 bis $1945-175$

\section{Jens Ebert}

Sprechende Wände. Häftlingsinschriften im Gefängnis Leistikowstraße Potsdam. Hrsg. von Ines Reich und Maria Schultz — 650

\section{Alexander Querengässer}

Stadt und Krieg. Leipzig in militärischen Konflikten vom Mittelalter bis ins 20. Jahrhundert. Hrsg. von Ulrich von Hehl - 170

Christian Koller

Nicholas Stargardt, Der deutsche Krieg 1939-1945 — 599

Heinz Stübig

Reinhard Stauber, Der Wiener Kongress — 227

Winfried Heinemann

Martin Steinacher, Maurice Bavaud - verhinderter Hitler-Attentäter im Zeichen des katholischen Glaubens? — 279

\section{Bruno Thoß}

Rolf Steininger, Deutschland und die USA. Vom Zweiten Weltkrieg bis zur Gegenwart — 626

\section{Andreas Lutsch}

Kristan Stoddart, The Sword and the Shield. Britain, America, NATO, and Nuclear Weapons, 1970-1976 - 312 
Hans Hecker

Arnold Suppan, Hitler - Beneš - Tito. Konflikt, Krieg und Völkermord in

Ostmittel- und Südosteuropa, 3 Bde -180

Dieter Hartwig

Tomas Termote, Krieg unter Wasser. Unterseebootflottille

Flandern 1915-1918 - 570

Michael Epkenhans

To Crown the Waves. The Great Navies of the First World War. Ed. by Vincent P. O'Hara, W. David Dickson and Richard Worth — 568

Andreas Himmelsbach

Leonie Treber, Mythos Trümmerfrauen. Von der Trümmerbeseitigung in der Kriegs- und Nachkriegszeit und der Entstehung eines deutschen Erinnerungsortes -629

Hans Hecker

Stefan Troebst, Erinnerungskultur - Kulturgeschichte - Geschichtsregion.

Ostmitteleuropa in Europa — 177

Dieter Hartwig

Lutz Unterseher, Antifritz. Hommage an Prinz Heinrich von Preußen 528

Denis Strohmeier

Die Verfolgung und Ermordung der europäischen Juden durch das nationalsozialistische Deutschland 1933-1945, Bd 7: Sowjetunion mit annektierten Gebieten I. Besetzte sowjetische Gebiete unter deutscher Militärverwaltung, Baltikum und Transnistrien. Bearb. von Bert Hoppe und Hildrun Glass 282

Peter Andreas Popp

Brian E. Vick, The Congress of Vienna. Power and Politics after Napoleon — 229

Christian Senne

André Vogel und Uwe Klar, Brennpunkt »Ostwall«. Die Kämpfe um die Festungsfront Oder-Warthe-Bogen im Winter $1945-617$ 


\section{Winfried Heineman}

Ilse-Margret Vogel, Über Mut im Untergrund. Eine Erzählung von Freundschaft, Anstand und Widerstand im Berlin der Jahre 1943-1945. Hrsg. von Jutta Hercher und Barbara Schieb 280

\section{Rüdiger Overmans}

Die Welt der Lager. Zur »Erfolgsgeschichte« einer Institution. Hrsg. von Bettina Greiner und Alan Kramer — 513

\section{Dieter Hartwig}

Stefan Wendt, Höhe 41. Krieg und Kriegsspiele an der Kieler Förde im 19. und 20. Jahrhundert -511

\section{Wolfgang Eisert}

Rüdiger Wenzke, Nationale Volksarmee - Die Geschichte. Unter Mitarb. von Torsten Diedrich und Denis Strohmeier — 644

Winfried Heinemann

Winfried Meyer, Klatt. Hitlers jüdischer Meisteragent gegen Stalin:

Überlebenskunst in Holocaust und Geheimdienstkrieg — 609

\section{Clemens Vollnhals}

Ernst Würzburger, „Der letzte Landsberger«. Amnestie, Integration und die Hysterie um die Kriegsverbrecher in der Adenauer-Ära — 634

Hans Hecker

Adam Zamoyski, 1815. Napoleons Sturz und der Wiener Kongress — 224

\section{Rüdiger Bergien}

Benjamin Ziemann, Veteranen der Republik. Kriegserinnerungen und demokratische Politik 1918-1933 — 261

Winfried Heinemann

Dirk Ziesing, Mit Gott für König und Vaterland. Geschichte des 1. Westfälischen Landwehr-Infanterie-Regiments 1813-1815 — 547

Mitarbeiterinnen und Mitarbeiter $-322,660$ 